\title{
Diverse antiviral lgG effector activities are predicted by unique biophysical antibody features
}

\author{
Hao D. Cheng 1,2, Karen G. Dowell ${ }^{3}$, Chris Bailey-Kellogg ${ }^{3}$, Brittany A. Goods ${ }^{4,5}$, J. Christopher Love ${ }^{4,5}$, \\ Guido Ferrari ${ }^{6,7}$, Galit Alter $^{8}$, Johannes Gach ${ }^{9}$, Donald N. Forthal ${ }^{9}$, George K. Lewis ${ }^{10}$, Kelli Greene ${ }^{6}$, \\ Hongmei Gao ${ }^{6}$, David C. Montefiori ${ }^{6,7}$ and Margaret E. Ackerman ${ }^{1,2^{*}}$ (D)
}

\begin{abstract}
Background: The critical role of antibody Fc-mediated effector functions in immune defense has been widely reported in various viral infections. These effector functions confer cellular responses through engagement with innate immune cells. The precise mechanism(s) by which immunoglobulin $\mathrm{G}(\mathrm{lgG})$ Fc domain and cognate receptors may afford protection are poorly understood, however, in the context of HIV/SHIV infections. Many different in vitro assays have been developed and utilized to measure effector functions, but the extent to which these assays capture distinct antibody activities has not been fully elucidated.

Results: In this study, six Fc-mediated effector function assays and two biophysical antibody profiling assays were performed on a common set of samples from HIV-1 infected and vaccinated subjects. Biophysical antibody profiles supported robust prediction of diverse lgG effector functions across distinct Fc-mediated effector function assays. While a number of assays showed correlated activities, supervised machine learning models indicated unique antibody features as primary contributing factors to the associated effector functions. Additional experiments established the mechanistic relevance of relationships discovered using this unbiased approach.

Conclusions: In sum, this study provides better resolution on the diversity and complexity of effector function assays, offering a clearer perspective into this family of antibody mechanisms of action to inform future HIV-1 treatment and vaccination strategies.
\end{abstract}

Keywords: IgG, Antibody, Effector function, HIV, Vaccine

\section{Background}

Passive administration of broadly neutralizing antibodies (bnAb) against HIV-1 has been shown to protect humanized mice [1], non-human primates (NHP) [2], and to reduce risk of infection by neutralization-sensitive viral strains in humans [3]. Robust protection conferred by broadly neutralizing antibodies has been widely

\footnotetext{
*Correspondence: Margaret.e.ackerman@dartmouth.edu

${ }^{1}$ Thayer School of Engineering, Dartmouth College, Hanover, NH, USA

Full list of author information is available at the end of the article
}

recognized as the consequence of IgG variable domain $(\mathrm{Fv})$ interactions with viral epitopes, via multiple mechanisms, including the block of viral attachment [4], inhibition of cell-cell transmission [5], and suppression of viremia [6]. The crystallizable fragment (Fc) part of the immunoglobulin (IgG) molecule, however, provides in vivo anti-viral effects as well, through engagement of innate immune $\mathrm{Fc} \gamma$ receptors (FcyRs). These activities contribute to bnAb-mediated anti-viral activity in some [7-10], but not all [11] studies that have looked original author(s) and the source, provide a link to the Creative Commons licence, and indicate if changes were made. The images or other third party material in this article are included in the article's Creative Commons licence, unless indicated otherwise in a credit line to the material. If material is not included in the article's Creative Commons licence and your intended use is not permitted by statutory regulation or exceeds the permitted use, you will need to obtain permission directly from the copyright holder. To view a copy of this licence, visit http://creativecommons.org/licenses/by/4.0/. The Creative Commons Public Domain Dedication waiver (http://creativecommons.org/publicdomain/zero/1.0/) applies to the data made available in this article, unless otherwise stated in a credit line to the data. 
to quantitatively evaluate their contribution, as recently reviewed [12].

Similarly, increasing evidence from animal models suggests that antibody effector functions also play a critical role in protective vaccine-mediated immunity. These activities include antibody-dependent phagocytosis [13], complement-dependent cytotoxicity (CDC) [14], antibody-dependent cellular viral inhibition (ADCVI) [15], and antibody-dependent cellular cytotoxicity (ADCC) [16], among others. Correlations between plasma ADCC activity and challenge resistance or decreased viral load have been observed in a number of NHP studies [17-20]. Antibody-dependent phagocytosis activity has also been reported to correlate with protection from infection in a growing number of preclinical studies [21, 22]. Polyfunctional antibody responses - those exhibiting the ability to elicit the anti-viral activities of diverse effector cell subsets, have also been reported to correlate with protection in vaccine studies [23]. The most definitive work to date that these correlations may represent mechanisms of protection comes from a passive transfer experiment in which polyfunctional but non-neutralizing antibodies were shown to protect NHP from SIV infection [24].

In humans, early work demonstrated the enrichment of ADCC-inducing antibodies in long-term HIV non-progressors [25-34]. Antibodies from HIV elite controllers have exhibited potentiated viral inhibition, NK activation, phagocytosis and antibody-dependent cellular cytotoxicity $[35,36]$. Moreover, immune-escape variants have been identified on ADCC epitopes, suggesting significant immune pressure applied from ADCC responses [37], which has also been supported in animal models [38, 39]. Diverse vaccine regimens have likewise supported a role of antibodies with potent effector function in accomplishing protection from infection [15]. In the context of low HIV-specific IgA, ADCC activity was associated with reduced risk of infection among RV144 HIV-1 vaccine recipients [40]. Additional analyses of this trial have observed that polyfunctional antibodies were induced [41]. Antibodies to the V1V2 variable loops [42] that elicited complement deposition [43], and IgG3 antibodies [44], which show elevated phagocytic activity [45], were also correlated with reduced risk of infection. Consistent with recent NHP studies [21, 22], the phagocytic activity of HIV-specific antibodies, along with their binding to FcyRIIa, was observed to correlate with reduced risk of infection in a phase $2 b$ trial (HVTN505) of a DNA + rAd5 vaccine expressing Envelope glycoproteins from clades A, B and C [46]. Excitingly, as in the pox-prime, protein boost RV144 vaccine, HIV-specific IgG3 responses were also associated with reduced risk of infection in this vaccine regimen [46].
While this may be rich evidence base on its face, it has been built on numerous distinct in vitro effector function assays developed to evaluate ADCC, ADCP, ADCVI and CDC activities. These assays differ in terms of the viral targets-ranging from recombinant antigen, to cells expressing envelope, to native virus; they differ in the effector cell populations employed-varying from purified to mixed populations of primary cells to tumorderived cell lines; and they diverge in terms of activities read out-consisting of surrogate measures of transcriptional changes, other effector cell phenotypes, or direct cytolytic or virus inhibition activity [47]. Insights into which assays best reflect processes relevant to outcomes in vivo principally stand on correlative evidence, and the antibody features that underlie potent activity in each assay are poorly resolved. To address this last limitation, we have combined here a high throughput, multiplexed assessment of features of potential importance to these functions with machine learning in order to define aspects of the polyclonal antibody response associated with each of six effector activities previously determined in a systematic evaluation of four ADCC, one ADCVI, and two phagocytosis assays performed on a common set of serum samples from infected and vaccinated individuals [48].

\section{Methods \\ Antibody samples}

As previously described [48], serum antibodies were purified from a total of 130 subjects, including treated chronically HIV-1 infected subjects $(\mathrm{n}=31)$, untreated chronically HIV-1 infected subjects $(n=28)$, elite controllers $(\mathrm{n}=31)$, who are individuals that are able to suppress HIV-1 replication in the absence of antiretroviral therapy [49], and recipients of HIV-1 vaccine AIDSVAX B/B gp120 $(n=20)$ or placebo $(n=10)$ [50]. Study subjects' clinical data are summarized in Additional file 1: Table S1. Polyclonal serum IgG antibody was separated from other serum proteins using the Melon gel IgG purification kit (ThermoFisher Scientific), as previously described [36]. Studies were approved by appropriate local Institutional Review Boards and each subject gave written informed consent.

\section{Effector functional assays}

Six IgG effector functional assays were performed in three different laboratories. A common stock of research reagents, antigens, and cryopreserved PBMCs were provided to each laboratory unless otherwise specified. Assay signal to noise was defined as the ratio of the average assay signal observed for serum in HIV-1 infected subjects to that observed for placebo recipients. 


\section{GTL ADCC}

The GranToxiLux (GTL) ADCC assay was performed as previously reported [51]. CEM.NKR $\mathrm{CCR}$ target cells were coated with the recombinant BaL HIV-1 gp120 antigen, followed by the labeling with GranToxiLux (GTL; Oncolmmunin, Inc), which fluoresces upon cleavage by Granzyme B (GrB) released by effector cells present in heterologous $\mathrm{PBMC}$ from a healthy donor. ADCC-mediating antibodies in IgG from serum samples were quantified as the percent of antigen-coated cells that took up GrB. Peak activity and the area under the titration curve (AUC) was reported as the assay readouts.

\section{LUC ADCC}

LUC ADCC assay was performed essentially as previous publication discussed $[52,53]$. A vector expressing both the BaL envelope (HIV BaL.LucR.T2A.ecto/293T/17; accession number DQ318211) and the Renilla luciferase reporter gene was used to transfect the CEM.NKR $\mathrm{CCR}_{\text {5 }}$ cell line. PBMCs were used as effector cells as previously described [54]. IgG was incubated with transfected cells and PBMCs for $6 \mathrm{~h}$. ADCC-mediating antibodies induced target cell death and resulted in the reduction of virus-derived luciferase signal, which was then reported as the assay readout. Peak activity and the area under the titration curve (AUC) were reported.

\section{RFADCC}

The rapid fluorescent ADCC (RFADCC) was performed as previously described [16]. Briefly, the CEM-NKR $\mathrm{CCR}_{5}$ $\mathrm{T}$ cell line was labeled with intracellular Carboxyfluorescein succinimidyl ester (CFSE), orange fluorescent dye PKH26, and recombinant SF162 gp120 protein. NK cells enriched from healthy donor blood with RosetteSep (Stem Cell Technologies) were used as effector cells. IgG was incubated with target cells and NK cells for $4 \mathrm{~h}$ at $37^{\prime} \mathrm{C}$. The proportion of PKH26+/CFSE- cells was determined by the flow cytometry and reported.

\section{BVADCC}

The bound virion ADCC (BVADCC) was performed as previously reported $[55,56]$. In brief, the CEM-NKR$\mathrm{CCR} 5+\mathrm{T}$ cell line was labeled with intracellular Carboxyfluorescein succinimidyl ester (CFSE), membrane orange fluorescent dye PKH26, and spinoculated with entry competent but replication-defective AT-2 BaL virions (Dr. Jeff Lifson, FCRC, NIH) [57-59] at $12{ }^{\circ} \mathrm{C}$ followed by incubation with serum IgG for $3 \mathrm{~h}$ at $37^{\circ} \mathrm{C}$. Dual dye loss of the target cells was recorded by the flow cytometry [16]. Peak activity and the area under the titration curve (AUC) were reported.

\section{ADCVI}

The antibody-dependent cell-mediated virus inhibition (ADCVI) assay was performed as previously reported [27]. ADCVI measures the change of p24 production in the presence of antibody and FcyR-bearing effector cells. Briefly, antibody was incubated with HIV-infected CEM$\mathrm{NKR}_{\text {CCR } 5}$ cells and fresh PBMCs for 7 days at $37^{\circ} \mathrm{C}$. The average percent decrease of p24 produced by infected cells was measured by p24 ELISA and defined as ADCVI Activity.

\section{Virion phagocytosis}

Phagocytosis was performed as previously described [60]. In brief, serum IgG was incubated with FITClabeled HIV-1 US657 (GenBank U04908), followed by the addition of THP-1 effector cells. FITC-labeled virus internalized by effector cells were identified by flow cytometry. The percentage of FITC + cells was multiplied by the corresponding fluorescent intensity and reported as the assay readout. The assay background, determined by calculating the FITC + cells in the absence of Envspecific antibody, was subtracted from the signals. The average readout of replicates was reported as an assay summary measure. Although phagocytosis assays using FITC-labeled virus may be useful for assessing Fc-Fc $\gamma R$ interactions, FITC has been shown to aggregate virions independently of antibody, raising some questions as to physiological relevance of this assay [61].

\section{IgG titering antigen array assay}

As previously described [62], a panel of HIV-1 antigens (Additional file 1: Table S2) was printed onto glass slides in triplicate, followed by incubation with blocking reagents. IgG was diluted in a ratio between 1:100 and 1:500 and then incubated with the array. Arrays were blocked again and washed thoroughly before labeling with fluorescent Goat Anti-human IgG detection antibody. The arrays were then detected by a GenePix 4200Al (Molecular Devices) and analyzed by GenePix Pro 6.0 (Molecular Devices). The median fluorescence intensity (MFI) of each spot was recorded, and the average of the triplicates was reported as assay summary measure.

\section{Fc array}

A customized multiplexed microsphere assay to define the characteristics of both Fv and Fc domains of purified serum antibody samples was conducted as previously described method [63]. Briefly, recombinant protein antigens (Additional file 1: Table S2) were covalently coupled to fluorescently-coded magnetic microspheres, 
which were incubated with dilute antibody to permit antigen binding, followed by washing and characterization of Fc domains using fluorescent detection reagents that included Fc receptor (FcR) tetramers, lectins, and secondary reagents (Additional file 1: Table S2) to identify total antibody isotypes and IgG subclasses. Data was acquired on a Bio-plex array reader (FlexMap 3D, BioPlex Manager 5.0, Bio-Rad). The net median fluorescence intensity (MFI) was reported.

\section{Data visualizations}

HIV-specific antibody features defined by the Fc Array were centered and scaled. Heatmap analysis of relationships between subjects and among antibody features defined by the Fc Array and antibody effector functions was performed using the heatmap.2 function [64] in $R$ 3.3.1 [65]. Similarities and differences in overall HIV-specific antibody response profiles defined by the Fc Array were defined by Uniform Manifold Approximation and Projection (UMAP) analysis using with the "UMAP" package [66, 67] in R.4.0.4 with default "naïve" method settings. For each subject, the two embedding coordinates were plotted using Graphpad Prism.

\section{Modeling antibody functions}

Effector function data collected from HIV-1 infected subjects (elite controllers, and chronically infected subjects on or off therapy) were predicted by biophysical data using HIV specific antigens (gp140, gp120, gp41, nonenvelope antigens and assessment of total serum IgG, IgA, and IgM) using a regularized random forest (RRF) tree algorithm [68]. Decision trees (2000) were trained to minimize regularized mean squared error and evaluated in the setting of fivefold cross-validation with the coefficient of regularization set at 0.8 . Prediction accuracy was computed as the Pearson correlation coefficient between mean predicted values and experimentally observed values. Contributions of individual biophysical antibody features to prediction of functional activities were evaluated by defining the percent increase in mean square error (\% Increase MSE) when individual features were permuted. Features with larger values represent those with greater importance/lower redundancy.

Model robustness was evaluated by comparison of performance of models trained with the experimental effector function data to those trained on permuted data [69], or with influenza-specific antibody response data. Influenza-specific antibody features were selected for use in this control as like HIV, influenza virus demonstrates a high level of antigenic diversity and all subjects were expected to be seropositive. In permutation tests, the model's output values (observed effector function) were randomized and RRF models were learned under the same settings using the same input features but permuted outputs, and this process was repeated for 250 iterations. The average prediction accuracy for both permuted outputs, and for influenza-based inputs was compared to the original predictions using an unpaired two-sided student $t$ test corrected for multiple hypothesis testing by controlling the false discovery rate to $1 \%$ using the two-stage step-up procedure of Benjamini, Krieger, and Yekutieli [70].

\section{Results}

Purified polyclonal antibodies collected from chronically infected subjects on (treated) and off (untreated) anti-retroviral therapy, elite controllers, and VAX004 HIV-1 recombinant gp120 vaccine and placebo recipients were previously tested in six different IgG effector function assays conducted by different labs [48]. These assays include four antibody-dependent cellular cytotoxicity assays (GTL, LUC, RFADCC, and BVADCC), one antibody-dependent phagocytosis (Phagocytosis) assay and one antibody-dependent cell-mediated virus inhibition (ADCVI) assay. As previously reported, serum IgG from HIV-infected subjects generally showed a more robust capacity to induce diverse effector functions than that from vaccine recipients, who in turn showed elevated responses as compared to placebo recipients (Fig. 1A). A subset of HIV-infected subjects showed elevated activity across multiple ADCC assays (RFADCC, BVADCC, and GTL). Different subsets of infected subjects showed high activity in ADCVI and Phagocytosis assays. As previously reported [71], serum IgG from a subset of the elite controllers exhibited a polyfunctional profile.

The same set of purified IgG samples were also tested in two different biophysical binding assays: a microarray approach used to detect IgG responses to a set of printed antigens, and a multiplexed bead array used to define antibody isotypes, subclasses, and FcyR- and lectin-ligation profiles across diverse antigen types and viral strains. To gain a sense of the specific features that differed between subjects and groups, HIV-specific response data from the bead array was visualized in clustered heatmap form (Fig. 1B). Whereas responses among placebo recipients were uniformly low across measurements, HIV-specific antibody responses among vaccine recipients and infected subjects varied considerably. Robust induction of IgG4 rarely resulted from natural infection, but was widely observed among VAX004 vaccine recipients. A subset of subjects exhibited antibodies with elevated ability to bind to lectins. This characteristic was not well correlated with other measures of the antibody response, suggesting it represents a unique distinction, the precise origin and functional consequences of which are unknown. Whereas gp41-specific responses were 


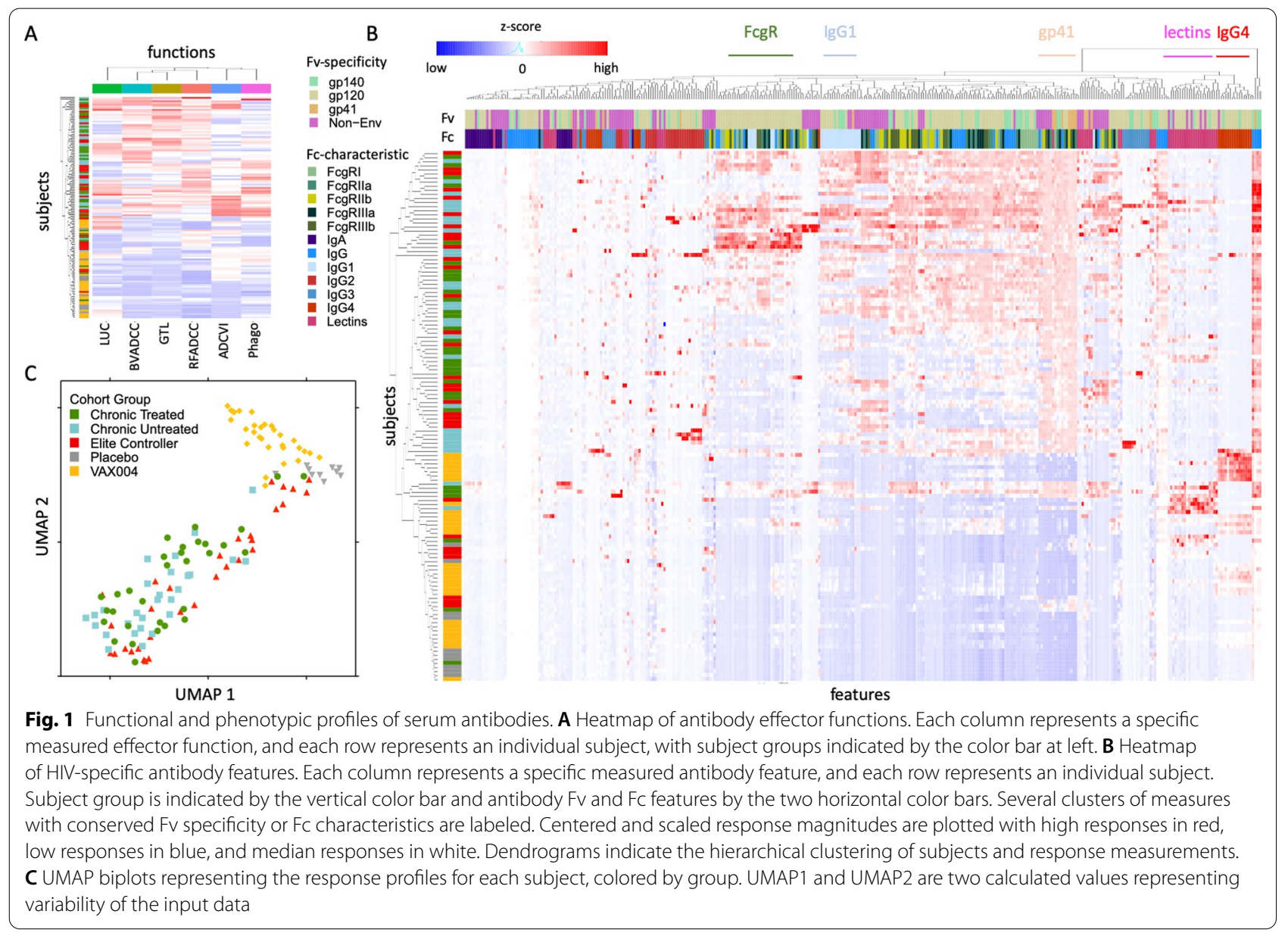

relatively similar in magnitude across infected subjects, responses to gp120 and gp140 showed a considerably greater degree of variability. Robust IgG2 responses were rare, and the overall magnitude of IgG antibodies was similar to IgG1 levels and antibody Fc $\gamma$ R-binding activity. Antibodies to internal proteins, including p24, nef, integrase, and others, were also observed among infected subjects.

To better represent these complex profiles graphically, dimensionality reduction by unsupervised UMAP analysis was performed across features specific to domains of HIV-1 envelope relevant to both vaccine and infection cohorts (gp120 and gp140 only) (Fig. 1C). Whereas placebo recipients were relatively tightly clustered, other groups showed greater spread in the distribution of subjects. Antibody profiles from VAX004 recipients were clearly distinct from those observed among infected subjects. Whether treated or untreated with antiviral therapy, chronically infected subjects fell in overlapping regions of the biplot, with elite controllers appearing somewhat shifted toward the lower right edge of this distribution.
The varying antibody responses observed among groups and between individuals provided the opportunity to attempt to define which antibody features were associated with each antibody effector function among HIV-infected subjects. To accomplish this goal, a supervised RRF machine learning model was trained to predict measured effector functions using IgG biophysical measurements. Predictive performance in the setting of fivefold cross-validation was evaluated across 20 replicates. Whereas models trained on actual data achieved robust prediction, defined as the degree of correlation between observed and predicted activity, models trained on permuted data had no predictive value (Fig. 2A). Similarly, when influenza rather than HIV-1 antibody features were used to train the model, accuracy was significantly degraded, most often to that expected at random (Fig. 2B). While all six IgG effector functions were robustly predicted, model accuracy varied considerably. This variation was strongly associated with the signal to noise profiles inherent to each assay in this data set (Fig. 2C). 

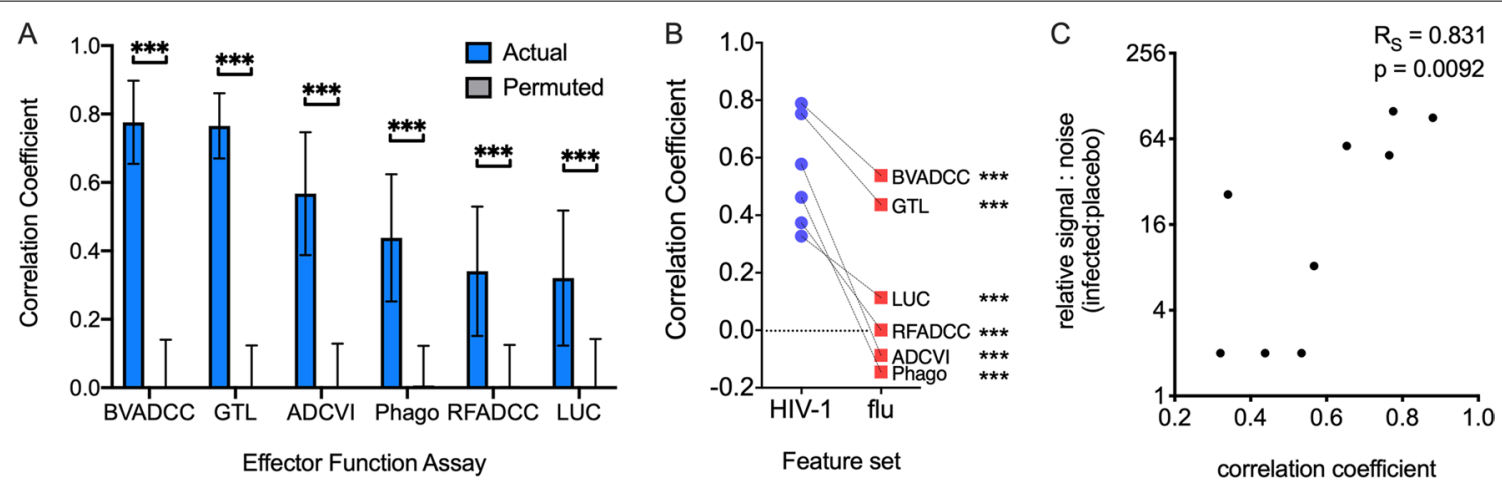

Fig. 2 IgG effector functions are robustly predicted by HIV-specific antibody biophysical features. A Correlation coefficients between observed values and predicted values resulting from models learned from actual (blue) and permuted (gray) data when HIV-specific antibody biophysical features were used to predict effector functions via a regularized random forest tree model in the setting of repeated cross-validation. Bars and whiskers denote the mean and standard deviation. B Accuracy of models learned from HIV-specific antibody features (blue) as compared to influenza-specific antibody features (red). Statistical significance was defined by t-test adjusted for multiple hypothesis testing $\left.{ }^{* * *} p<0.001\right)$. C. Scatterplot of prediction quality (correlation coefficient) and assay signal to noise ratio. Spearman correlation coefficient and statistical significance reported in inset

The degree of agreement between predicted and actual activity in each assay for each HIV-infected subject group suggested that, in general, models predicted activity among each group equivalently well (Fig. 3). Having established robust predictive performance across assays and subject groups, we next sought to define the features supporting each model (Fig. 4). The relative importance of contributing features, whether they represented a strictly quantitative (IgG titer) or qualitative (Ig subclasses or Fc domain characteristics) aspect of the humoral response, was defined for each function. Across effector assays, distinctions in the antigen-specificity and Fc domain characteristics relevant to each activity were readily apparent.

GTL and BVADCC assays were the most accurately predicted, and both aim to measure antibody-mediated cellular cytotoxicity. While they were among the most highly correlated pairs of assays, the major supporting features turned out to be very different. For GTL assay activity, gp120-specific antibody ligation of Fc $\gamma$ RIII features were primary supporting features, consistent with the reliance of this assay on gp120-coated target cells, and NK cells as effectors. In contrast, a broader diversity of envelope-specific antibody ligation of FcyRII contributed primarily to the prediction of BVADCC results, and only three FcyRIII features were selected among the top 30 features. In terms of antigen recognition, given the use of inactivated virions in this assay, contributions from gp41- and gp140-specific antibodies are likely to have direct mechanistic relevance. In terms of Fc domain characteristics, a strong dependence on FcyRII, more widely associated with phagocytosis and a diversity of effector cells, was noted. Interestingly, subsequent experiments have demonstrated that this assay, which employs unfractionated PBMCs, actually primarily assesses activity mediated by monocytes, and which is mechanistically dependent on FcyRII [72, 73].

Though not predicted as well as these other ADCC assays, models of RFADCC assay activity showed a strong dependence on FcyRIII, consistent with the use of purified NK cells as effectors, and its readout of cell permeabilization dependent on GrB activity driven by Fc $\gamma$ RIII ligation. One attribute common to each of these assays is their strong reliance on the measures of antibody quality (FcR-related features), as compared to measures of response magnitude alone (anti-human IgG detection reagents), indicating that titer-related measurements were less important than Fc-specific measurements in predicting these functions.

For ADCVI and phagocytosis assays, p24-specific antibodies were major contributing features. While both assays evaluated responses in the context of replication-competent virus, meaning that p24 was present, it is not clear how a mechanistic role for $\mathrm{p} 24$-specific antibodies in restricting viral outgrowth (ADCVI) or driving phagocytosis (fluorescent virion uptake) might be mediated. For ADCVI, which showed a clear degradation in model accuracy when p24 features were omitted $\left(R_{P}=0.55\right.$ versus 0.47 without $p 24$ features, $\mathrm{p}<0.05$ ), one possibility raised to by this model is that p24-specific antibodies present in each sample may interfere with the p24 quantitation that serves as the measure of virus replication read out by the assay. This possibility does not exist for the phagocytosis assay, which does not involve p24 quantitation, and for which omission of $\mathrm{p} 24$ features still resulted in a small 


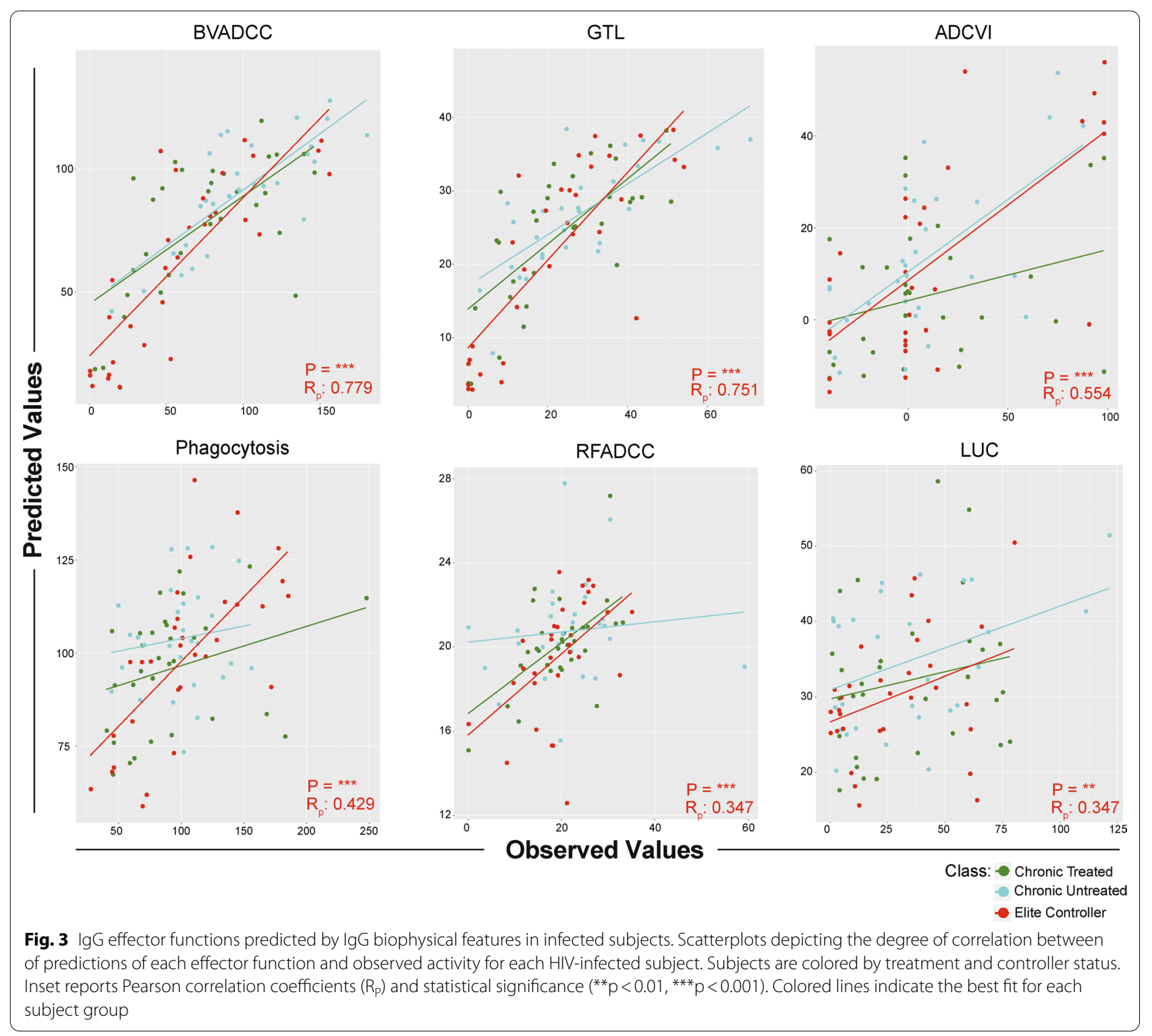

but statistically significantly reduction in accuracy $\left(R_{P}=0.43\right.$ versus 0.39 without $p 24$ features, $\left.p=0.10\right)$. Interestingly, and unlike other functions, model performance for phagocytosis was superior among elite controllers (elite controllers $R_{P}=0.66, p=0.00006$; chronic treated $R_{P}=0.06, p=0.05$; chronic untreated $\left.R_{P}=0.16, p=0.4\right)$, who are known to exhibit higher levels of p24-specific antibodies [74, 75] as compared to chronically infected subject groups. Overall, while direct mechanistic contributions cannot be determined without further experiment, the data driven approach employed linked the otherwise unrelated observations of better ADCVI activity observed in elite controllers [36] and long term non-progressors [25] and their elevated p24-specific antibody levels [74, 75]. Coupled to observations related p24-specific responses to protection made in the setting of protective vaccines [76], these results suggest a potential avenue for further mechanistic evaluation [77].

Lastly, led by a titer measurement (IgG specific to gp120 CM), a number of envelope-specific Fc $\gamma$ RIII features contribute to predictions in the LUC assay. Intriguingly, despite the use of IgA depleted samples, a number of IgA features, including responses specific to internal proteins such as Nef and Integrase also made contributions. While mechanistic contributions cannot be excluded, interpretation of model features must also consider assay reproducibility and model performance 


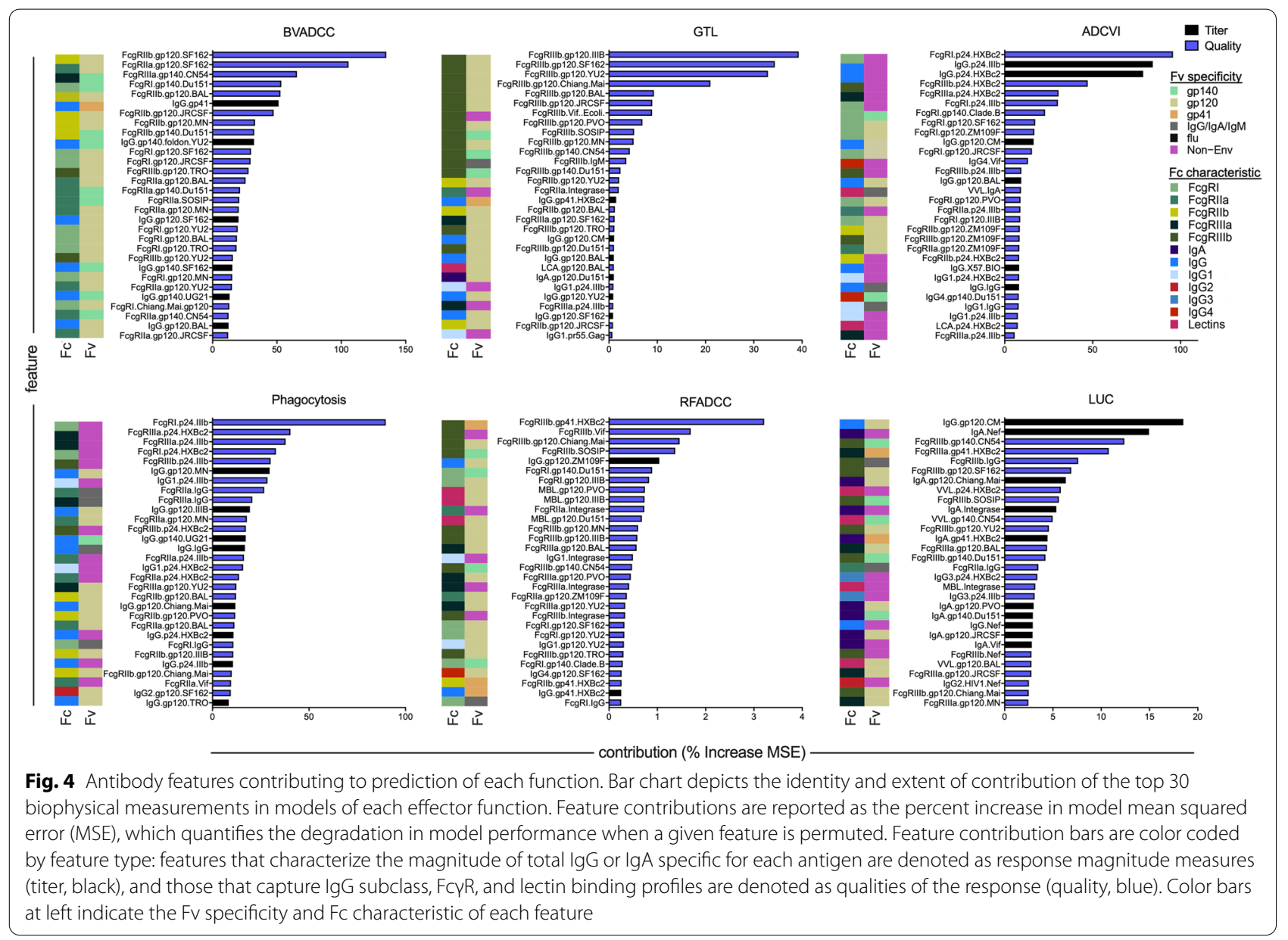

quality. The best performing models provided new, mechanistically relevant insights, and the less well-performing models can be considered to point to both the limitations of modeling noisy biological data, as well as to the complexity of antibody effector functions and the challenges to developing robust in vitro assays for their assessment.

\section{Discussion}

Titers of antigen-specific binding antibodies and neutralizing antibodies are often useful but not entirely sufficient measures to fully explain therapeutic benefit or vaccine efficacy across a wide variety of pathogens [76, 78-84]. Given the challenges in achieving high titers of broadly neutralizing antibodies, the discrepancy between antibody quantity and antibody quality have posed special challenges to development of an effective HIV-vaccine. Because both NHP and human immune correlate studies of experimental vaccines have suggested the importance of antibody effector functions [46, 85-87], these functions offer an additional means to restrict infection, thereby motivating interest in defining how different aspects of antibody quality and quantity contribute to diverse activities. Potent effector functions are associated with a set of unique antibody characteristics, including $\mathrm{N}$-linked glycosylation of the Fc domain, antibody titer, immune complex size/geometry, epitope specificity, binding avidity, and IgG subclasses [56, 88-92].

While these in vitro antibody activities have shown associations with protection from HIV-1 infection, slower progression of AIDS, or lower viral loads in both humans and nonhuman primates, most of the current effector function assays utilize pre-defined antigens and cell lines, which do not fully recapitulate the complexity of the in vivo immune responses. The variation of individual effector cell populations and genetic polymorphisms in Fc receptors all pose additional challenges to the interpretation of results in simplified in vitro antibody effector function assays. These and other hostspecific factors add a layer of further functional diversity. Despite the inability of correlative relationships to provide direct evidence of mechanism of action, it may be important to apply multiple and orthogonal assays to 
acquire a comprehensive understanding of the spectrum of possible in-vivo antiviral antibody activities.

Here, to dissect this rich landscape, supervised machine learning models were trained to predict effector function among HIV-infected subjects using antibody profiling data. The two most accurately predicted effector function assays, GTL, and BVADCC, were highly correlated with each other, but predicted by a distinct set of response features. In general, these and other functional assays chiefly relied upon measures of antibody quality ( $\mathrm{F} c \gamma \mathrm{R}$ binding propensity and IgG subclass) rather than antibody quantity (antigen-specific IgG titer), and exposed the role of an unanticipated receptor in one of the assays that was validated experimentally. Collectively, consistent with prior studies [75, 76, 91, 93], these results emphasize the value of specific quality attributes of humoral responses in inducing potent effective effector functions.

While there was considerable diversity in the antibody profiles among infected subjects, responses in vaccinees were even more distinct. In particular, IgG4 responses were strikingly elevated among VAX004 vaccine recipients as compared to responses observed among infected individuals, as was previously noted for VAX003 [44]. Such profound differences point to the plasticity of the immune response. Better understanding of how class switch recombination is regulated has clear value to both vaccine design and development of novel approaches to antibody-driven allergic and autoimmune responses.

\section{Conclusions}

Given the growing number of studies in the past decade that have revealed the essential role of Fc and FcyR-mediated effector activities in both animal and human studies [12], coupled to the diversity of assays used to characterize these activities [47], we sought to marry previously published results for a set of cell-based FcyR-mediated effector function assays [48] to biophysical antibody profiling assays on a common set of serum samples from HIV infected and vaccinated individuals. Intrigued by acquiring a deeper resolution of specific antibody features associated with complementary effector functions, this study discovered more differences than similarities among the different assays. By using machine learning methods to relate activity observed in each assay to underlying characteristics of the humoral response as defined by two high-throughput, multiplexed profiling assays, we found that assays with good signal to noise could be robustly predicted from HIV- but not permuted or influenza-specific antibody profile data. Further, even among the most highly correlated assays, predictions were driven by unique combinations of response characteristics that could be mechanistically confirmed. While these results do not enable identification of the activities most relevant to antibodymediated protection from HIV infection in vivo, they offer further insights into the diversity of effector cell types and associated antibody phenotypes that may contribute to viral blockade and restriction.

\section{Supplementary Information}

The online version contains supplementary material available at https://doi. org/10.1186/s12977-021-00579-9.

Additional file 1: Table S1. Clinical characteristics of HIV+ Subject Groups. Mean and interquartile ranges (IQR) of ages, sex, viral load, and CD4 T cell counts for untreated, treated, and elite controllers. Presence or absence of protective HLA-B alleles were balanced across groups. Table S2. Antigen specificities and Fc detection reagents.

\section{Acknowledgements}

VAX004 samples were provided by Global Solutions for Infectious Disease.

\section{Authors' contributions}

HDC, KGD, and CBK analyzed and interpreted data. BAG and JG generated and interpreted data. $\mathrm{KG}, \mathrm{HG}$, and DCM oversaw creation and implementation of the study plan and sample sharing. CBK, JCL, GF, GA, DNF, GKL, and MEA oversaw experiments, and analyzed and interpreted data. HDC and MEA drafted the manuscript. All authors critically read and approved the manuscript.

\section{Funding}

These studies were supported by the Bill and Melinda Gates Foundation's Collaboration for AIDS Vaccine Discovery (CAVD) Grants OPP1032817, OPP1114729, OPP1032144, OPP1033109, and NIAID R01 Al131975 and P01 Al120756.

Availability of data and materials

The datasets analyzed in this study are available from the corresponding author on reasonable request.

\section{Declarations}

\section{Ethics approval and consent to participate}

Studies were approved by appropriate local Institutional Review Boards and each subject gave written informed consent.

\section{Consent for publication}

Not applicable.

\section{Competing interests}

The authors report no competing interests.

\section{Author details}

${ }^{1}$ Thayer School of Engineering, Dartmouth College, Hanover, NH, USA. ${ }^{2}$ Molecular and Cellular Biology Program, Dartmouth College, 14 Engineering Dr., Hanover, NH 03755, USA. ${ }^{3}$ Department of Computer Science, Dartmouth College, Hanover 03755, USA. ${ }^{4}$ Department of Chemical Engineering, Massachusetts Institute of Technology, Cambridge, MA 02139, USA. ${ }^{5}$ Department of Biological Engineering, Koch Institute at MIT, Massachusetts Institute of Technology, Cambridge, MA 02139, USA. ${ }^{6}$ Department of Surgery, Duke University Medical Center, Durham, NC 27710, USA. ${ }^{7}$ Duke Human Vaccine Institute, Duke University Medical Center, Durham, NC 27719, USA. ${ }^{8}$ Ragon Institute of MGH, MIT, and Harvard, Cambridge, MA 02139, USA. ${ }^{9}$ Division of Infectious Diseases, Irvine School of Medicine, University California, Irvine, CA 92697, USA. ${ }^{10}$ Division of Vaccine Research, Institute of Human Virology, University Maryland School of Medicine, Baltimore, MD 21201, USA. 
Received: 22 June 2021 Accepted: 20 October 2021

Published online: 30 October 2021

\section{References}

1. Balazs AB, Chen J, Hong CM, et al. Antibody-based protection against HIV infection by vectored immunoprophylaxis. Nature. 2012;481(7379):81-4.

2. Mascola JR, Stiegler G, VanCott TC, et al. Protection of macaques against vaginal transmission of a pathogenic HIV-1/SIV chimeric virus by passive infusion of neutralizing antibodies. Nat Med. 2000;6(2):207-10.

3. Corey L, Gilbert PB, Juraska M, et al. Two randomized trials of neutralizing antibodies to prevent HIV-1 acquisition. N Engl J Med. 2021;384(11):1003-14.

4. Ugolini S, Mondor I, Parren PW, et al. Inhibition of virus attachment to CD4+ target cells is a major mechanism of T cell line-adapted HIV-1 neutralization. J Exp Med. 1997;186(8):1287-98.

5. Reh L, Magnus C, Schanz M, et al. Capacity of broadly neutralizing antibodies to inhibit HIV-1 cell-cell transmission is strain- and epitopedependent. PLoS Pathog. 2015;11(7):e1004966.

6. Caskey M, Klein F, Lorenzi JCC, et al. Viraemia suppressed in HIV1 -infected humans by broadly neutralizing antibody 3BNC117. Nature. 2015;522(7557):487-91.

7. Bournazos S, Klein F, Pietzsch J, et al. Broadly neutralizing anti-HIV-1 antibodies require Fc effector functions for in vivo activity. Cell. 2014;158(6):1243-53.

8. Asokan M, Dias J, Liu C, et al. Fc-mediated effector function contributes to the in vivo antiviral effect of an HIV neutralizing antibody. Proc Natl Acad Sci USA. 2020;117(31):18754-63.

9. Hessell AJ, Hangartner L, Hunter M, et al. Fc receptor but not complement binding is important in antibody protection against HIV. Nature. 2007:449(7158):101-4.

10. Wang P, Gajjar MR, Yu J, et al. Quantifying the contribution of Fc-mediated effector functions to the antiviral activity of anti-HIV-1 IgG1 antibodies in vivo. Proc Natl Acad Sci USA. 2020;117(30):18002-9.

11. Parsons MS, Lee WS, Kristensen AB, et al. Fc-dependent functions are redundant to efficacy of anti-HIV antibody PGT121 in macaques. J Clin Investig. 2019;129(1):182-91.

12. Carpenter MC, Ackerman ME. Recent insights into Fc-mediated effector responses to HIV-1. Curr Opin HIV AIDS. 2020;15(5):282-9.

13. Ackerman ME, Moldt B, Wyatt RT, et al. A robust, high-throughput assay to determine the phagocytic activity of clinical antibody samples. J Immunol Methods. 2011;366(1-2):8-19.

14. Hezareh M, Hessell AJ, Jensen RC, et al. Effector function activities of a panel of mutants of a broadly neutralizing antibody against human immunodeficiency virus type 1. J Virol. 2001;75(24):12161-8.

15. Forthal DN, Gilbert PB, Landucci G, et al. Recombinant gp120 vaccineinduced antibodies inhibit clinical strains of HIV-1 in the presence of Fc receptor-bearing effector cells and correlate inversely with HIV infection rate. J Immunol. 2007;178(10):6596-603.

16. Gomez-Roman VR, Florese RH, Patterson $\sqcup$, et al. A simplified method for the rapid fluorometric assessment of antibody-dependent cell-mediated cytotoxicity. J Immunol Methods. 2006;308(1-2):53-67.

17. Asmal M, Sun Y, Lane S, et al. Antibody-dependent cell-mediated viral inhibition emerges after simian immunodeficiency virus SIVmac251 infection of rhesus monkeys coincident with gp140-binding antibodies and is effective against neutralization-resistant viruses. J Virol. 2011;85(11):5465-75.

18. Felber BK, Lu Z, Hu X, et al. Co-immunization of DNA and protein in the same anatomical sites induces superior protective immune responses against SHIV challenge. Cell Rep. 2020;31(6):107624.

19. Florese RH, Demberg T, Xiao P, et al. Contribution of nonneutralizing vaccine-elicited antibody activities to improved protective efficacy in rhesus macaques immunized with Tat/Env compared with multigenic vaccines. J Immunol. 2009;182(6):3718-27.

20. Hidajat R, Xiao P, Zhou Q, et al. Correlation of vaccine-elicited systemic and mucosal nonneutralizing antibody activities with reduced acute viremia following intrarectal simian immunodeficiency virus SIVmac251 challenge of rhesus macaques. JVirol. 2009;83(2):791-801.
21. Ackerman ME, Das J, Pittala S, et al. Route of immunization defines multiple mechanisms of vaccine-mediated protection against SIV. Nat Med. 2018:24(10):1590-8.

22. Om K, Paquin-Proulx D, Montero M, et al. Adjuvanted HIV-1 vaccine promotes antibody-dependent phagocytic responses and protects against heterologous SHIV challenge. PLoS Pathog. 2020;16(9):e1008764.

23. Barouch $\mathrm{DH}$, Alter $\mathrm{G}$, Broge $\mathrm{T}$, et al. Protective efficacy of adenovirus/ protein vaccines against SIV challenges in rhesus monkeys. Science. 2015;349(6245):320-4.

24. Alter G, Yu WH, Chandrashekar A, et al. Passive transfer of vaccineelicited antibodies protects against SIV in rhesus macaques. Cell. 2020;183(1):185-96 e14.

25. Baum LL, Cassutt KJ, Knigge K, et al. HIV-1 gp120-specific antibodydependent cell-mediated cytotoxicity correlates with rate of disease progression. J Immunol. 1996;157(5):2168-73.

26. Ahmad R, Sindhu ST, Toma E, et al. Evidence for a correlation between antibody-dependent cellular cytotoxicity-mediating anti-HIV-1 antibodies and prognostic predictors of HIV infection. J Clin Immunol. 2001;21(3):227-33.

27. Forthal DN, Landucci G, Daar ES. Antibody from patients with acute human immunodeficiency virus (HIV) infection inhibits primary strains of HIV type 1 in the presence of natural-killer effector cells. J Virol. 2001;75(15):6953-61.

28. Stratov I, Chung A, Kent SJ. Robust NK cell-mediated human immunodeficiency virus (HIV)-specific antibody-dependent responses in HIVinfected subjects. J Virol. 2008;82(11):5450-9.

29. Chung AW, Navis M, Isitman G, et al. Activation of NK cells by ADCC antibodies and HIV disease progression. J Acquired Immune Deficiency Syndr. 2011;58(2):127-31.

30. Johansson SE, Rollman E, Chung AW, et al. NK cell function and antibodies mediating ADCC in HIV-1-infected viremic and controller patients. Viral Immunol. 2011;24(5):359-68.

31. Wren LH, Chung AW, Isitman G, et al. Specific antibody-dependent cellular cytotoxicity responses associated with slow progression of HIV infection. Immunology. 2013;138(2):116-23.

32. Lambotte $\mathrm{O}$, Ferrari G, Moog C, et al. Heterogeneous neutralizing antibody and antibody-dependent cell cytotoxicity responses in HIV-1 elite controllers. AIDS. 2009;23(8):897-906.

33. Lambotte O, Pollara J, Boufassa F, et al. High antibody-dependent cellular cytotoxicity responses are correlated with strong CD8 T cell viral suppressive activity but not with B57 status in HIV-1 elite controllers. PLoS ONE. 2013;8(9):e74855.

34. Forthal DN, Landucci G, Haubrich R, et al. Antibody-dependent cellular cytotoxicity independently predicts survival in severely immunocompromised human immunodeficiency virus-infected patients. J Infect Dis. 1999;180(4):1338-41.

35. Ackerman ME, Dugast AS, McAndrew EG, et al. Enhanced phagocytic activity of HIV-specific antibodies correlates with natural production of immunoglobulins with skewed affinity for FcyR2a and FcyR2b. J Virol. 2013;87(10):5468-76.

36. Ackerman ME, Crispin M, Yu X, et al. Natural variation in Fc glycosylation of HIV-specific antibodies impacts antiviral activity. J Clin Investig. 2013;123(5):2183-92.

37. Chung AW, Isitman G, Navis M, et al. Immune escape from HIV-specific antibody-dependent cellular cytotoxicity (ADCC) pressure. Proc Natl Acad Sci USA. 2011;108(18):7505-10.

38. Horwitz JA, Bar-On Y, Lu CL, et al. Non-neutralizing antibodies alter the course of HIV-1 infection in vivo. Cell. 2017;170(4):637-48 e10.

39. Santra S, Tomaras GD, Warrier R, et al. Human non-neutralizing HIV-1 envelope monoclonal antibodies limit the number of founder viruses during SHIV mucosal infection in rhesus macaques. PLoS Pathog. 2015;11(8):e1005042.

40. Haynes BF, Gilbert PB, McElrath MJ, et al. Immune-correlates analysis of an HIV-1 vaccine efficacy trial. N Engl J Med. 2012;366(14):1275-86.

41. Chung AW, Ghebremichael M, Robinson H, et al. Polyfunctional Fceffector profiles mediated by $\lg \mathrm{G}$ subclass selection distinguish RV144 and VAX003 vaccines. Sci TransI Med. 2014;6(228):228ra38.

42. Zolla-Pazner S, deCamp A, Gilbert PB, et al. Vaccine-induced lgG antibodies to V1V2 regions of multiple HIV-1 subtypes correlate with decreased risk of HIV-1 infection. PLoS ONE. 2014;9(2):e87572. 
43. Perez LG, Martinez DR, deCamp AC, et al. V1V2-specific complement activating serum IgG as a correlate of reduced HIV-1 infection risk in RV144. PLOS ONE. 2017;12(7):e0180720.

44. Yates NL, Liao H-X, Fong Y, et al. Vaccine-induced Env V1-V2 lgG3 correlates with lower HIV-1 infection risk and declines soon after vaccination. Sci Transl Med 2014;6(228):228ra39-ra39.

45. Chu TH, Crowley AR, Backes I, et al. Hinge length contributes to the phagocytic activity of HIV-specific lgG1 and IgG3 antibodies. PLoS Pathog. 2020;16(2):e1008083.

46. Neidich SD, Fong Y, Li SS, et al. Antibody Fc effector functions and lgG3 associate with decreased HIV-1 risk. J Clin Investig. 2019;129(11):4838-49.

47. Lewis GK, Ackerman ME, Scarlatti G, et al. Knowns and unknowns of assaying antibody-dependent cell-mediated cytotoxicity against HIV-1. Front Immunol. 2019;10:1025

48. Huang $Y$, Ferrari $G$, Alter $G$, et al. Diversity of antiviral lgG effector activities observed in HIV-infected and vaccinated subjects. J Immunol. 2016;197(12):4603-12.

49. Pereyra F, Addo MM, Kaufmann DE, et al. Genetic and immunologic heterogeneity among persons who control HIV infection in the absence of therapy. J Infect Dis. 2008;197(4):563-71.

50. Flynn NM, Forthal DN, Harro CD, et al. Placebo-controlled phase 3 trial of a recombinant glycoprotein 120 vaccine to prevent HIV-1 infection. J Infect Dis. 2005;191(5):654-65

51. Pollara J, Hart L, Brewer F, et al. High-throughput quantitative analysis of HIV-1 and SIV-specific ADCC-mediating antibody responses. Cytometry Part A J Int Soc Anal Cytol. 2011;79(8):603-12.

52. Pollara J, Bonsignori M, Moody MA, et al. HIV-1 vaccine-induced $\mathrm{C} 1$ and V2 Env-specific antibodies synergize for increased antiviral activities. J Virol. 2014;88(14):7715-26.

53. Edmonds TG, Ding H, Yuan $X$, et al. Replication competent molecular clones of HIV-1 expressing Renilla luciferase facilitate the analysis of antibody inhibition in PBMC. Virology. 2010;408(1):1-13.

54. Sambor A, Garcia A, Berrong M, et al. Establishment and maintenance of a PBMC repository for functional cellular studies in support of clinical vaccine trials. J Immunol Methods. 2014;409:107-16.

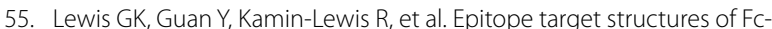
mediated effector function during HIV-1 acquisition. Curr Opin HIV AIDS 2014:9(3):263-70.

56. Guan Y, Pazgier M, Sajadi MM, et al. Diverse specificity and effector function among human antibodies to HIV-1 envelope glycoprotein epitopes exposed by CD4 binding. Proc Natl Acad Sci U S A. 2013;110(1):E69-78.

57. Rossio JL, Esser MT, Suryanarayana K, et al. Inactivation of human immunodeficiency virus type 1 infectivity with preservation of conformational and functional integrity of virion surface proteins. J Virol. 1998;72(10):7992-8001.

58. Chertova E, Bess JW Jr, Crise BJ, et al. Envelope glycoprotein incorporation, not shedding of surface envelope glycoprotein (gp120/SU), Is the primary determinant of SU content of purified human immunodeficiency virus type 1 and simian immunodeficiency virus. J Virol. 2002;76(11):5315-25.

59. Chertova E, Crise BJ, Morcock DR, et al. Sites, mechanism of action and lack of reversibility of primate lentivirus inactivation by preferential covalent modification of virion internal proteins. Curr Mol Med. 2003;3(3):265-72.

60. Forthal DN, Landucci G, Bream J, et al. FcgammaRlla genotype predicts progression of HIV infection. J Immunol. 2007;179(11):7916-23.

61. Gach JS, Bouzin M, Wong MP, et al. Human immunodeficiency virus type-1 (HIV-1) evades antibody-dependent phagocytosis. PLoS Pathog. 2017;13(12):e1006793.

62. Han Q, Bagheri N, Bradshaw EM, et al. Polyfunctional responses by human $T$ cells result from sequential release of cytokines. Proc Natl Acad Sci U S A. 2012;109(5):1607-12.

63. Brown EP, Dowell KG, Boesch AW, et al. Multiplexed Fc array for evaluation of antigen-specific antibody effector profiles. J Immunol Methods. 2017:443:33-44

64. Warnes $\mathrm{GR}$, Bolker B, Bonebakker $\mathrm{L}$, et al. gplots: various $\mathrm{R}$ programming tools for plotting data. 2015.

65. R: A language and environment for statistical computing. R Foundation for Statistical Computing. 2013.

66. Konopka T. umap: Uniform Manifold Approximation and Projection. 2020.
67. McInnes L, Healy J, Melville J. UMAP: Uniform Manifold Approximation and Projection for Dimension Reduction. arXiv. 2020;1802.03426.

68. Liaw A, Wiener M, Breiman L, et al. RRF: feature selection with regularized random forest. 2018.

69. Ernst MD. Permutation methods: a basis for exact inference. Stat Sci. 2004;19(4):676-85.

70. Benjamini Y, Krieger AM, Yekutieli D. Adaptive linear step-up procedures that control the false discovery rate. Biometrika. 2006;93(3):491-507.

71. Ackerman ME, Mikhailova A, Brown EP, et al. Polyfunctional HIV-specific antibody responses are associated with spontaneous HIV control. PLOS Pathog. 2016;12(1):e1005315.

72. Kramski M, Schorcht A, Johnston AP, et al. Role of monocytes in mediating HIV-specific antibody-dependent cellular cytotoxicity. J Immunol Methods. 2012:384(1-2):51-61.

73. Pollara J, Orlandi C, Beck C, et al. Application of area scaling analysis to identify natural killer cell and monocyte involvement in the GranToxiLux antibody dependent cell-mediated cytotoxicity assay. Cytometry Part A J Int Soc Anal Cytol. 2018;93(4):436-47.

74. French MA, Center RJ, Wilson KM, et al. Isotype-switched immunoglobulin G antibodies to HIV Gag proteins may provide alternative or additional immune responses to "protective" human leukocyte antigen-B alleles in HIV controllers. AIDS. 2013;27(4):519-28.

75. Alter G, Dowell KG, Brown EP, et al. High-resolution definition of humoral immune response correlates of effective immunity against HIV. Mol Syst Biol. 2018;14(3):e7881.

76. Pittala S, Bagley K, Schwartz JA, et al. Antibody Fab-Fc properties outperform titer in predictive models of SIV vaccine-induced protection. Mol Syst Biol. 2019;15(5):e8747.

77. Chung AW, Mabuka JM, Ndlovu B, et al. Viral control in chronic HIV-1 subtype $C$ infection is associated with enrichment of p24 lgG1 with FC effector activity. AIDS. 2018;32(10):1207-17.

78. Kazmin D, Nakaya HI, Lee EK, et al. Systems analysis of protective immune responses to RTS, S malaria vaccination in humans. Proc Natl Acad Sci U S A. 2017;114(9):2425-30

79. Blaney JE, Marzi A, Willet M, et al. Antibody quality and protection from lethal Ebola virus challenge in nonhuman primates immunized with rabies virus based bivalent vaccine. PLoS Pathog. 2013;9(5):e1003389-e.

80. Fox JM, Roy V, Gunn BM, et al. Optimal therapeutic activity of monoclonal antibodies against chikungunya virus requires Fc-FcgammaR interaction on monocytes. Sci Immunol. 2019. https://doi.org/10.1126/sciimmunol. aav5062.

81. Li D, He W, Liu X, et al. A potent human neutralizing antibody Fc-dependently reduces established HBV infections. Elife. 2017. https://doi.org/10. 7554/eLife.26738.

82. Tortorici MA, Beltramello M, Lempp FA, et al. Ultrapotent human antibodies protect against SARS-CoV-2 challenge via multiple mechanisms. Science. 2020:370(6519):950-7.

83. Ilinykh PA, Huang K, Santos Rl, et al. Non-neutralizing antibodies from a Marburg infection survivor mediate protection by Fc-effector functions and by enhancing efficacy of other antibodies. Cell Host Microbe. 2020;27(6):976-91 e11.

84. Winkler ES, Gilchuk P, Yu J, et al. Human neutralizing antibodies against SARS-CoV-2 require intact Fc effector functions for optimal therapeutic protection. Cell. 2021;184(7):1804-20 e16.

85. Rolland M, Edlefsen PT, Larsen BB, et al. Increased HIV-1 vaccine efficacy against viruses with genetic signatures in Env V2. Nature. 2012;490(7420):417-20.

86. Prentice HA, Tomaras GD, Geraghty DE, et al. HLA class II genes modulate vaccine-induced antibody responses to affect HIV-1 acquisition. Sci Transl Med. 2015:7(296):296ra112.

87. Li SS, Gilbert PB, Tomaras GD, et al. FCGR2C polymorphisms associate with HIV-1 vaccine protection in RV144 trial. J Clin Investig. 2014;124(9):3879-90

88. Lux A, Nimmerjahn F. Impact of differential glycosylation on lgG activity. Adv Exp Med Biol. 2011;780:113-24.

89. Strohmeier GR, Brunkhorst BA, Seetoo KF, et al. Neutrophil functional responses depend on immune complex valency. J Leukoc Biol. 1995;58(4):403-14.

90. Schroeder HW, Cavacini L. Structure and function of immunoglobulins. J Allergy Clin Immunol. 2010;125(2):S41-52. 
91. Chung AW, Crispin M, Pritchard L, et al. Identification of antibody glycosylation structures that predict monoclonal antibody Fc-effector function. AIDS. 2014;28(17):2523-30.

92. Boesch AW, Osei-Owusu NY, Crowley AR, et al. biophysical and functional characterization of rhesus macaque lgG subclasses. Front Immunol. 2016;7:589.

93. Lai Jl, Licht AF, Dugast AS, et al. Divergent antibody subclass and specificity profiles but not protective HLA-B alleles are associated with variable antibody effector function among HIV-1 controllers. J Virol. 2014;88(5):2799-809.

\section{Publisher's Note}

Springer Nature remains neutral with regard to jurisdictional claims in published maps and institutional affiliations.
Ready to submit your research? Choose BMC and benefit from:

- fast, convenient online submission

- thorough peer review by experienced researchers in your field

- rapid publication on acceptance

- support for research data, including large and complex data types

- gold Open Access which fosters wider collaboration and increased citations

- maximum visibility for your research: over $100 \mathrm{M}$ website views per year

At BMC, research is always in progress.

Learn more biomedcentral.com/submissions 